\title{
Neoplastic transformation by TERT in FGF-2-expanded human mesenchymal stem cells
}

\author{
EMI YAMAOKA ${ }^{1,2}$, EISO HIYAMA ${ }^{1,2}$, YUSUKE SOTOMARU ${ }^{1}$, YOSHIYUKI ONITAKE ${ }^{2}$, \\ IKUKO FUKUBA $^{2}$, TAKESHI SUDO ${ }^{2}$, TAIJIRO SUEDA ${ }^{2}$ and KEIKO HIYAMA ${ }^{1}$ \\ ${ }^{1}$ Natural Science Center for Basic Research and Development, ${ }^{2}$ Department of Surgery, \\ Graduate School of Biomedical Science, Hiroshima University, Hiroshima, Japan
}

Received January 28, 2011; Accepted April 4, 2011

DOI: 10.3892/ijo.2011.1029

\begin{abstract}
The low percentage of human mesenchymal stem cells (hMSCs) in bone marrow necessitates their in vitro expansion prior to clinical use in regenerative medicine. We evaluated the effect of long-term culture of hMSCs on telomere length and transformation capacity by TERT transfection. hMSCs were isolated from the bone marrow aspirates of 24 donors and cultured with fibroblast growth factor-2 (FGF-2). Six cell lines with $>500$ population doubling levels were considered immortalized. TERT was transfected into two of the six lines for a comparison of telomere length, telomerase activity, differential capacity, colony formation capacity in soft agar and tumorigenicity in immunodeficient (NOD-SCID) mice. hMSC lines exhibited elongated telomeres without the activation of telomerase and retained multi-lineage differentiation potential upon chondrogenic or adipogenic differentiation, while non-immortalized hMSCs showed a marked reduction in telomere length in the differentiation process. Immortalized hMSCs showed anchorage-independence and formed tumors in NOD-SCID mice. Histologically, these tumors consisted of differentiated cells such as fat tissue and cartilage. Two TERTtransfected hMSC lines showed high rates of tumor formation in NOD-SCID mice. These tumors were histologically similar to teratocarcinoma without differentiated cells. These cells may provide a model for the origin of cancer stem cells from adult stem cells, and indicate the possibility that telomerase activation has a major role in the malignant transformation of human stem cells. These data suggest that adult hMSCs have a potential for neoplastic transformation and have implications for the use of hMSCs in tissue engineering and regenerative medicine.
\end{abstract}

\section{Introduction}

Stem cells in various adult tissues play an important role in the regeneration of damaged tissue and maintenance of

Correspondence to: Dr Eiso Hiyama, Hiroshima University, 1-2-3 Kasumi, Minami-ku, Hiroshima 734-8551, Japan

E-mail: eiso@hiroshima-u.ac.jp

Key words: stem cell, telomerase, TERT, immortality, cancer homeostasis in the tissues in which they reside. Human mesenchymal stem cells (hMSCs) are of great interest in regenerative medicine because they have the potential to differentiate into a variety of cells such as osteoblasts, adipocytes, chondrocytes, myocytes, and probably neuronal cells (1-5). Because of these properties, hMSCs might be a potential resource for future cell therapy. The goal of this study is to establish clinical cell lines with long life spans that retain their parental properties. However, clinical application has been difficult due to problems with retaining enhanced viability during storage, isolating cell populations with specific criteria, and expanding an in vitro system to obtain a sufficient number of cells without affecting their genomic characteristics and differentiation properties.

At present, there is very little evidence of whether changes in these properties occur during expansion. Normal hMSCs have a limited replicative capacity of at most 40- to 50-population doubling levels (PDL). The clinical use of hMSCs requires a large number of cells. Fibroblast growth factor-2 (FGF-2) increases PDL in hMSC monolayer cultures while preserving the differentiation potential $(6,7)$. These FGF-2 expanded MSCs [FGF(+) hMSCs] exhibited long telomeres without upregulation of telomerase activity. Then, to elucidate the function of telomerase in hMSCs, we transfected TERT in these $\mathrm{FGF}(+)$ hMSC cell lines. In this study, we report on the immortalization of hMSCs by ALT (alternative lengthening of telomeres) and the neoplastic activity of these immortalized hMSCs following TERT transfection. The results indicate that immortalization might be regulated by ALT and that telomerase activation might be correlated with malignant transformation in hMSCs.

\section{Materials and methods}

Cell lines and TERT transfection. Human mesenchymal stem cells were isolated from bone marrow (BM) aspirates of 24 human donors ( 16 men and 8 women, from 16 to 64 years of age) after obtaining informed consent as previously described (7). Aspirates were cultured in high-glucose Dulbecco's modified Eagle's medium (DMEM, Invitrogen Corp., Carlsbad, CA, USA) containing 15\% heat-inactivated FBS (Sigma-Aldrich, St. Louis, MO, USA) and antimicrobial agents. After confirming cell adhesion, hMSCs were cultured with or without FGF-2 (final 
concentration $1 \mathrm{ng} / \mathrm{ml}$; R\&D Systems Inc., Minneapolis, MN, USA). After again reaching confluence, the cells were reseeded under the same conditions. Six samples proliferated for $>500$ PDL. These cells were transfected with the TERT expression plasmid hTERTn2 (provided by Professor F. Ishikawa). hMSCs were transfected by using TransIT ${ }^{\circledR}$-LT1 Reagent (Mirus Bio Corp., Madison, WI, USA) with $2 \mu \mathrm{g}$ of hTERTn2 linearized with $N r u$ I or with the vector plasmid linearized. G418 (Wako, Osaka, Japan) $(300 \mu \mathrm{g} / \mathrm{ml})$ was added to the medium after $24 \mathrm{~h}$ to select for clones harboring the transfected gene. G418 selection was continued for at least 1 month in exponentially growing cultures. hMSCs and TERT-transfected hMSCs were collected at various PDLs using trypsin followed by a rinse with PBS and were stored at $-80^{\circ} \mathrm{C}$.

Telomere length analysis. Telomere length was estimated using the length of terminal restriction fragments (TRFs) as measured by Southern blot analysis $(8,9)$. Genomic DNA was isolated using proteinase $\mathrm{K}$ followed by phenol/chloroform extraction. Extracted DNA was completely digested with HinfI, electrophoresed in a $0.6 \%$ agarose gel $(2 \mu \mathrm{g} /$ lane $)$, transferred to a nitrocellulose membrane, and hybridized with 5 '-end $\left[{ }^{32} \mathrm{P}\right]$-labeled (TTAGGG) $)_{4}$. The length of the peak signals measured by the Bioimage Analyzer, BAS-2000 (Fuji film, Kanagawa, Japan) was estimated as the length of TRFs. Digested DNA was also subjected to pulse-field gel electrophoresis for the evaluation of long TRFs.

Telomere length was also measured by a fluorescence in situ hybridization (FISH) technique known as TeloFISH. TeloFISH was performed using a fluorescein isothiocyanate (FITC)-labeled peptide nucleic acid (PNA) probe specific for (TTAGGG) $n$ sequences (Telomere PNA FISH Kit/FITC, Dako Cytomation Co., Kyoto, Japan). Briefly, hMSCs were treated with colcemid (10 $\mu \mathrm{g} / \mathrm{ml}$, Life Technologies) to prepare metaphase spreads and were pre-fixed with $2 \mathrm{ml}$ freshly made fixative $(3+1 \mathrm{v} / \mathrm{v}$ methanol/glacial acetic acid). After $10 \mathrm{~min}$ at room temperature, cells were centrifuged at $500 \mathrm{x} \mathrm{g}$ for $10 \mathrm{~min}$. This pre-fix treatment was performed three times. The fixed cells were fixed to the slides, denatured at $80^{\circ} \mathrm{C}$ for $3 \mathrm{~min}$, and hybridized with the FITC-conjugated telomere PNA probe for $30 \mathrm{~min}$. The slides were then rinsed and washed at $65^{\circ} \mathrm{C}$ for $5 \mathrm{~min}$ and counterstained with DAPI.

Assay for telomerase activity and detection for TERT. Extraction of telomerase protein and evaluation of its activity were performed using the telomeric repeat amplification protocol (TRAP) $(10,11)$. Briefly, $10^{5}-10^{6}$ cells were homogenized in CHAPS lysis buffer. After $30 \mathrm{~min}$ of incubation on ice, the levels of telomerase activity were measured using the TRAPeze XL Kit (Serological Co., Gaithersburg, MD, USA), which is a quantitative, fluorescently-labeled PCR system for the estimation of relative telomerase activity levels with the use of a PCR internal control. The levels of fluorescein and sulforhodamine in each PCR product were measured in a fluorescent plate reader (Wallac, Perkin-Elmer, Wellesley, MA, USA). The level of telomerase activity, expressed in units of total product generated (TPG), was quantified by the ratio of the fluorescein intensity of the entire TRAP ladder in each sample (which was corrected with respect to the negative control) to the sulfo- rhodamine intensity of the internal control (which was corrected with respect to background).

The expression levels of TERT in hMSCs were estimated by the reverse transcription-quantitative polymerase chain reaction. Total cellular RNA was extracted from tumor tissues by acid-guanidium-phenol chloroform method (12). First strand complementary DNA (cDNA) was synthesized with reverse transcriptase and random primers using a High-Capacity cDNA Archive Kit (Applied Biosystems, Foster City, CA). An aliquot of the cDNA (equivalent to $20 \mathrm{ng}$ total RNA) was subjected to real-time RT-PCR using the TaqMan Gene Expression Assay (Applied Biosystems) for TERT and Pre-Developed TaqMan Assay Reagents (Applied Biosystems) for $18 \mathrm{~S}$ as an internal control. Results of the three or more independent measurements were averaged, and the relative gene expression levels were calculated as ratios to $18 S$ expression levels for each sample.

Induction of chondrogenic, adipogenic, and osteogenic differentiation. Chondrogenic adipogenic and osteogenic differentiation potential were measured in hMSCs and TERTtransfected hMSCs. A modified version of Johnstone's pellet culture system was used to induce chondrogenesis. Cells $\left(10^{6}\right)$ were placed in a $15-\mathrm{ml}$ polypropylene tube (Greiner BioOne, Frickenhausen, Germany) and centrifuged. The pellet was cultured at $37^{\circ} \mathrm{C}$ in a humidified atmosphere of $95 \%$ air and $5 \% \mathrm{CO}_{2}$ in $1 \mathrm{ml}$ of chondrogenic medium containing highglucose DMEM (Invitrogen) supplemented with $10 \mathrm{ng} / \mathrm{ml}$ transforming growth factor (TGF)- $\beta 3$ (Sigma), $10^{-7} \mathrm{M}$ dexamethasone (Sigma), $50 \mu \mathrm{g} / \mathrm{ml}$ ascorbic acid-2-phosphate sesquimagnesium salt (Sigma), $40 \mu \mathrm{g} / \mathrm{ml}$ L-proline (Nacalai tesque, Kyoto, Japan), ITS-A supplement (Invitrogen; $10 \mu \mathrm{g} / \mathrm{ml}$ insulin, $6.7 \mathrm{ng} / \mathrm{ml}$ sodium selenite, $5.5 \mu \mathrm{g} / \mathrm{ml}$ transferrin, $110 \mu \mathrm{g} / \mathrm{ml}$ sodium pyruvate), and $1.25 \mathrm{mg} / \mathrm{ml}$ bovine serum albumin (BSA, Sigma). Adipogenic differentiation was assessed by incubating cells with Adipogenic Induction Medium (Cambrex Bio Sciences, Walkersville, MD, USA) and maintained in hMSC Adipogenic Maintenance SingleQuots (Cambrex Bio Sciences) for 2-3 weeks. Adipocytes are recognized by the accumulation of lipid-containing vacuoles that stain red with Oil Red-O. Osteogenic differentiation was assessed by incubating the cells with DMEM-LG and 10\% FBS supplemented with $0.1 \mu \mathrm{M}$ dexamethasone, $10 \mu \mathrm{M} \beta$-glycerophosphate, and $50 \mu \mathrm{M}$ ascorbate (all from Sigma-Aldrich) for 2-3 weeks. Cultures were stained with silver nitrate (von Kossa's staining) to assess mineralization.

Colony formation assay with soft agar. Anchorage-dependency of the cells was evaluated by conventional colony formation assay with soft agar. Trypsinized cells $\left(5 \times 10^{3}\right)$ were resuspended in DMEM containing 10\% FBS and $0.4 \%$ SeaPlaque GTG agarose (Bio-products) and poured onto bottom ager containing $10 \%$ FBS and $0.53 \%$ agarose in a $30-\mathrm{mm}$ culture dish. After 21 days of culture at $37^{\circ} \mathrm{C}$ with $5 \% \mathrm{CO}_{2}$, colony number was evaluated with crystal violet staining.

Transplantation of hMSCs in immunocompromised non-obese diabetic (NOD)-SCID mice. Tumor cells were resuspended in a 1:1 mixture of Matrigel (Becton-Dickinson) and serum-free medium (DMEM with $1 \%$ penicillin/streptomycin). A $100-\mu 1$ 
suspension containing between 100 and 100000 cells was injected subcutaneously (s.c.) into the flanks of 6- to 10-week-old male NOD/SCID immunodeficient mice obtained from CLEA Japan (Tokyo, Japan). If tumors developed, tumor cells were dispersed and subcutaneously injected into secondrecipient NOD-SCID mice. Animal studies were approved by the Institutional Animal Care and Use Committee (Hiroshima University IRB No. A06-43) and conducted according to the Institutional Guidelines of Hiroshima University.

A portion of each developed tumor was re-cultured in vitro under the same conditions as the original tumor cells. The remainder of the tumor was fixed in formalin for pathological examination. The expression of TERT was detected by immunohistochemistry using an affinity-purified polyclonal rabbit antibody against TERT (EST21A) (Alpha Diagnostic International, San Antonio, TX) as previously described (13).

Statistical analysis. The data are shown as the mean \pm SEM. Fisher's exact test was used for $2 \times 2$ contingency tables.

\section{Results}

Culture of hMSCs. Since hMSCs cultured without FGF-2 [MSCs-FGF(-)] entered senescence after approximately 20 PDLs, these hMSCs were cultured with FGF-2 [MSCs-FGF(+): FGF-2-expanded hMSCs]. FGF-2 was added to the culture at a final concentration of $1 \mathrm{ng} / \mathrm{ml}$; these cells achieved more than 100 PDLs, doubling four times over a 4-day period, and exhibited small spindle-shaped aspects (Fig. 1A). It was these small spindle-shaped cells that had a high proliferation potential (Fig. 1B). Among 24 hMSC clones cultured with FGF-2, 6 had high proliferative activity (>100 PDLs). These clones have approximately the same proliferative rate in DMEM with or without FGF-2, and were considered to be immortalized hMSC clones (Fig. 1C).

TERT or vector alone was transfected into these 6 clones after $100 \mathrm{PDL}$. These cells, which were obtained after selection with $300 \mu \mathrm{g} / \mathrm{ml}$ of $\mathrm{G} 418$, were similar to pre-transfection cells in proliferative activity and morphology. The cell lines proliferated indefinitely (>1000 PDL) without major morphological changes after transfection with TERT (Fig. 1B and C). These cell lines were used in all subsequent analyses.

Telomere length and telomerase in hMSCs and TERTtransfected hMSCs. Southern blot analysis showed that immortalized hMSCs maintain relatively long telomeric lengths $(>23 \mathrm{~kb})$. Pulse-field electrophoresis confirmed that the length of these elongated telomeres was approximately $40 \mathrm{~kb}$ (Fig. 2A). TeloFISH analysis also detected long telomeres in these expanded hMSCs and TERT-transfected hMSCs (Fig. 2B). However, there was no detectable telomerase activity in these hMSCs. Therefore, these findings suggest that long telomeres in hMSCs are maintained by a telomeraseindependent mechanism.

Telomere lengths in TERT-transfected hMSCs were similar to those in untransfected hMSCs. The TRAP assay detected telomerase activity in TERT-transfected hMSCs but none in vector-transfected cells. All six lines showed high telomerase activity (14.6-1349.2 TPG) at 1-month post-transfection. The six TERT-transfected hMSC clones were cultured for more

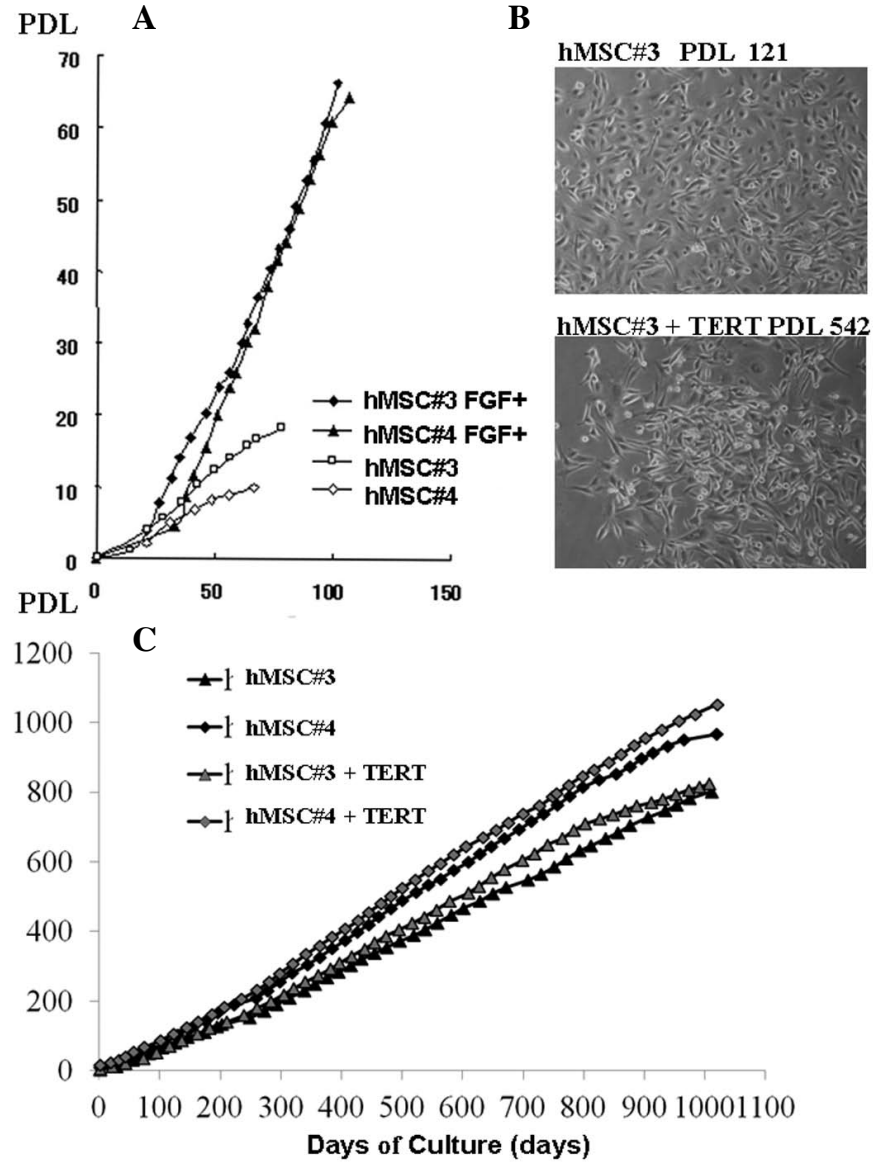

Figure 1. Cell proliferation and morphology of hMSCs. (A), Proliferation curve of $\mathrm{FGF}(+)$ hMSC or FGF(-) hMSC derived from bone marrow aspirates of normal human donors aged 32 (hMSC\#3), and 43 years (hMSC\#4) of age, respectively. After confirming cell adhesion to the plate, MSCs were cultured with $15 \%$ FBS-DMEM with or without FGF-2 (final concentration $1 \mathrm{ng} / \mathrm{ml}$ ). Cumulative population doublings level (PDL) is regarded as zero for culture starting immediately after the primary culture of cells, and calculated to increase according to the equation: $\log _{2}\{$ (the number of collected cells)/(the number of seeded cells) $\}$. After TERT was transfected into FGF(+) hMSCs after culture of the 100 PDL. (B), Representative morphologies of hMSC (hMSC\#3 PDL 121) and TERT-transfected hMSC (hMSC\#3 + TERT PDL 542). There are no morphological changes after TERT transfection. (C), Proliferation curve of hMSCs or TERT transfected hMSCs of 2 clones (hMSC\#3 and hMSC\#4). Cumulative PDL is zero immediately after the primary culture of cells, and is calculated according to the equation: $\log _{2}$ \{(the number of collected cells)/(the number of seeded cells) $\}$. There are no changes of proliferative activity after TERT transfection.

than 100 PDLs before telomerase activity was measured again. Telomerase activity was diminished in four clones (0.21.31 TPG in hMSC lines \#1, \#2, \#5, and \#6) and retained in two (1761.3 and 965.3 TPG in hMSC lines \#3 and \#4, respectively). TERT expression was also detected in these 6 clones just after transfection using RT-PCR, but retained in only two clones (hMSC\#3 and hMSC\#4) (Fig. 3). Among these 6 cell lines, we focused on the two clones with retained telomerase and TERT expression for the following examination.

In vitro differentiation potential in lineages of immortalized hMSC lines. hMSCs reportedly have an extensive potential to differentiate into multiple cell lineages including osteoblasts, chondrocytes, and adipocytes (14). To evaluate the effect of TERT transfection on differentiation, each hMSC line was 

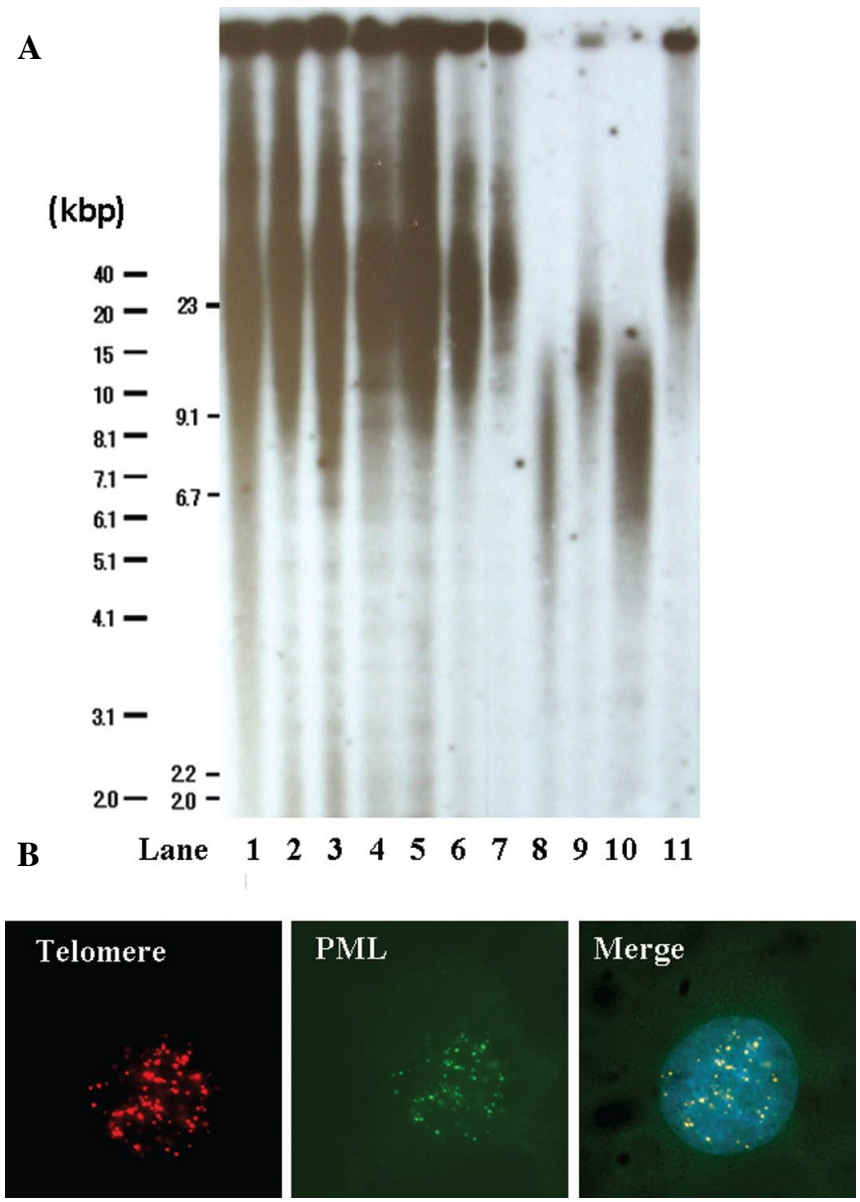

Figure 2. (A), Telomere lengths in hMSCs. Terminal restriction fragment lengths were measured by Southern blot analysis using pulse-field gel electrophoresis. Lane 1, hMSC\#3 (+FGF) 15.2 PDL; lane 2, hMSC\#3 (+FGF) 318.9 PDL; lane 3, hMSC\#3 (+FGF) 501.6 PDL; lane 4, hMSC\#4 (+FGF) 55 PDL; lane 5, hMSC\#4 (+FGF) 105.3 PDL; lane 6, hMSC\#4 (+FGF) 554.6 PDL; lane 7, TERT-transfected hMSC\#4 484.6 PDL; lane 8, hMSC\#3 (-FGF) 24.6 PDL; lane 9, hMSC\#3 (-FGF) 46.4 PDL; lane 10, hMSC\#4 (-FGF) 26.2 PDL; lane 11, TERT-transfected hMSC\#3 426.2 PDL. (B), Confocal imaging showing the nuclear expression patterns in the telomere (Cy3 labeled) and promyelocytic leukemia (PML) body-related antigen $\mathrm{mAb}$ (FITC labeled). hMSC\#3 (+FGF) 318.9 PDL demonstrated strong telomeric signals and large PML bodies. Both signals merged in the nuclei.

stimulated in lineage-specific induction medium for 2-4 weeks (Fig. 4). In adipocyte-specific culture medium, all of the cell lines accumulated lipid-rich vacuoles in their cytoplasm within 2 weeks, which were made evident by Oil Red-O staining. After 3 weeks in chondrocyte differentiation media, hMSCs and TERT-transfected hMSCs had metachromasia by TB and expression of type II collagen. After culturing cells as a micromass pellet for 21 days, the size of the TERT-transfected hMSCs was slightly smaller those of the parent hMSCs, but both had differentiated into cartilage (Fig. 4A) and, both hMSCs and TERT-transfected hMSCs showed greater adipogenetic ability (Fig. 4B). After 2 weeks in osteoblast induction medium, hMSCs and TERT-transfected hMSCs showed a marked increase in alkaline phosphatase expression (Fig. 4C), an osteoblast marker TERT-transfected immortalized mesenchymal stem cell lines retained the ability to differentiate into three lineages, although among cell lines there were significant variations in response to lineage-specific induction.
A

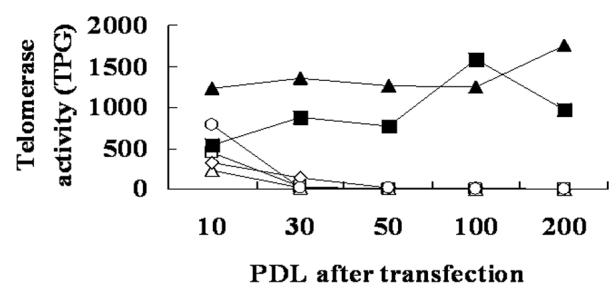

B

\begin{tabular}{|ll|}
\hline- hMSC\#1 & - hMSC\#2 \\
- hMSC\#3 & - hMSC\#4 \\
$\neg \sim$ hMSC\#5 & $-\curvearrowleft$ hMSC\#6 \\
\hline
\end{tabular}

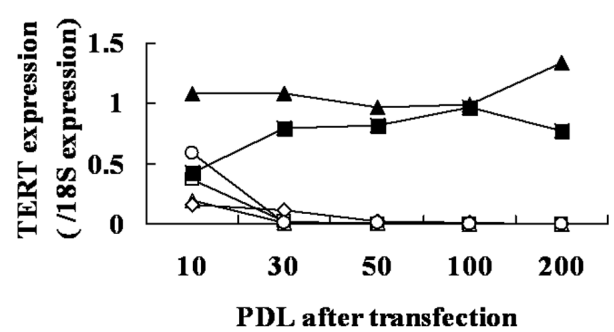

Figure 3. Telomerase activity and TERT expression in TERT transfected hMSCs (A), In the 6 TERT transfected clones, telomerase activity was diminished in four clones (0.2-1.31 TPG in hMSC lines \#1, \#2, \#5, and \#6) and retained in two (1761.3 and 965.3 TPG in hMSC lines \#3, and \#4, respectively). (B), TERT expression were also retained in these two clones but diminished in the remaining 4 clones.
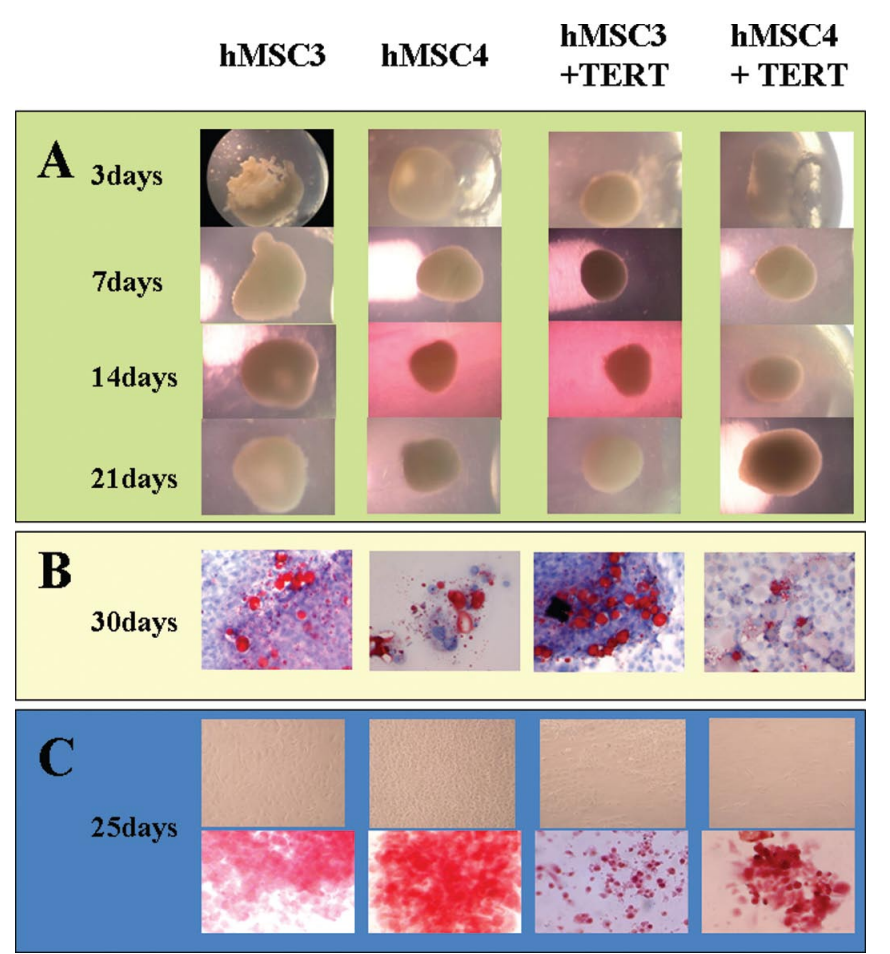

Figure 4. Induction of hMSC differentiation. (A), Macroscopic view of chondrogenesis. Pellet culture of FGF(+) MSC-derived pellets at 3, 7, 14, and 21 days. (B), Microscopic views of adipogenesis. Oil Red-O staining of FGF(+) MSC-derived pellets at 3, 7, 14, and 21 days. (C), Microscopic views of osteogenesis of hMSCs.

TERT enhanced the colony-forming ability of hMSCs. The colony formation assay revealed that colony numbers formed in soft agar were highly correlated with TERT expression levels in hMSCs. The colony numbers in TERT-transfected hMSC clones (hMSC\#3 and \#4) were significantly higher than 


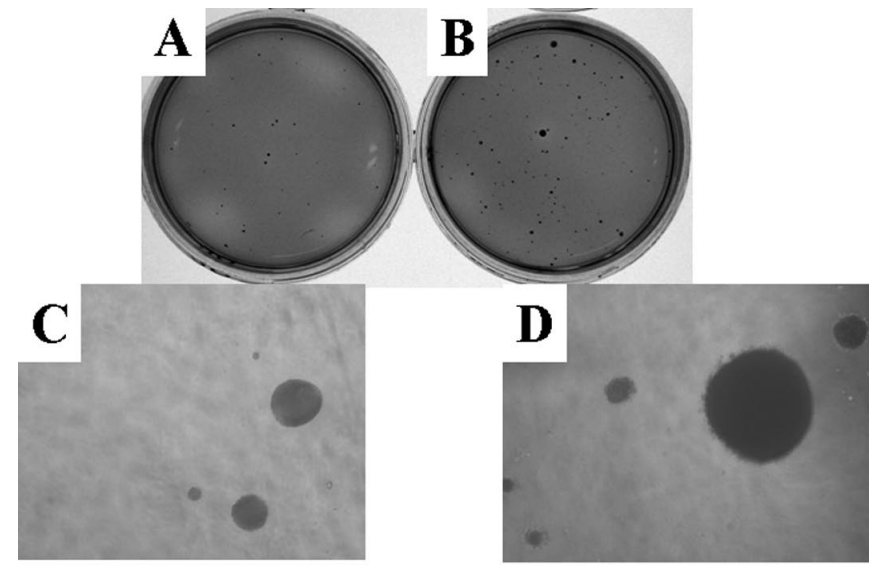

$\mathbf{E}$

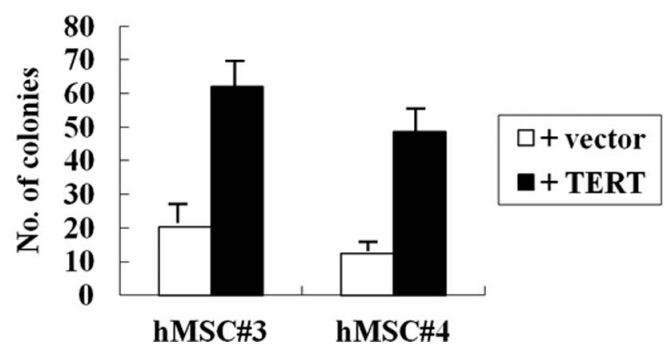

Figure 5. Soft agar assay of hMSCs colony numbers at day 21 in colony formation assay with soft agar for a vector-transfected clone (hMSC\#3+vector) (A) and TERT-transfected clone (hMSC\#3+TERT) (B). Both colony types were stained with crystal violet. Colonies formed from TERT-transfected clones (D) were significantly larger than those of the vector transfected clone (C). (E), Bar graph showed the difference of colony formation between the vector and TERT-transfected clones in hMSC\#3 and \#4.

those of hMSCs without detectable TERT expression $(\mathrm{P}<0.001)$. In addition, the cellular growth rates of TERT-transfected hMSC (hMSC\#3 and \#4) clones are higher than those of vectortransfected clones (Fig. 5).

In vivo tumorigenic assays. TERT-transfected hMSC cells $\left(10^{2}\right.$ or $\left.10^{5}\right)$ were subcutaneously injected into NOD-scid mice and tumor development was evaluated. As shown in Table I and Fig. 6, all mice injected with $10^{5}$ cells of TERT-expressing hMSC clones (TERT-hMSC\#3 and TERT-hMSC\#4) developed tumors and 5 of 8 mice injected with $10^{2}$ cells of these TERTexpressing hMSC clones developed tumors. In contrast, four other TERT-transfected hMSC clones (TERT-hMSC\#1, \#2, \#5, and \#6) and all the vector-transfected hMSCs, did not produce tumors after injections of $10^{2}$ cells, although an injection of two vector-transfected hMSCs clones (vectorhMSC\#2 and vector-hMSC\#3) and two TERT-transfected hMSC clones (TERT-hMSC\#2 and TERT-hMSC\#5) did induce tumorigenesis in mice after $10^{5}$ injection (Table I).

Macroscopically, tumors derived from TERT-expressing hMSCs (TERT-hMSC\#3 and TERT-hMSC\#4) were large and hard (Fig. 6B), while tumors arising from cells without TERT expression were small and relatively soft (Fig. 6F). Histological examination revealed that the former were teratocarcinomalike tumors (Fig. 6C and D), while the latter were teratomas with several differentiated tissues, including fat and bone (Fig. 6G and H). Immunohistological examination revealed
Table I. Xenograft and tumor growth in NOD/SCID injected with hMSCs.

\begin{tabular}{|c|c|c|}
\hline hMSCs & $\begin{array}{l}\text { No. of cells } \\
\text { injected }\end{array}$ & $\begin{array}{c}\text { Tumor formation } \\
\text { (tumor volume, } \mathrm{mm}^{3} \text { ) }\end{array}$ \\
\hline \multicolumn{3}{|c|}{ TERT-hMSCs } \\
\hline \multirow[t]{2}{*}{ \#1 } & 100 & $0 / 4$ \\
\hline & 100000 & $0 / 4$ \\
\hline \multirow[t]{2}{*}{$\# 2$} & 100 & $0 / 4$ \\
\hline & 100000 & $1 / 4(648)$ \\
\hline \multirow[t]{2}{*}{ \#3 } & 100 & $2 / 4(765,546)$ \\
\hline & 100000 & $4 / 4(8740,4080,3504,3360)$ \\
\hline \multirow[t]{2}{*}{$\# 4$} & 100 & $3 / 4(1728,1200,855)$ \\
\hline & 100000 & $4 / 4(12000,4560,3420)$ \\
\hline \multirow[t]{2}{*}{ \#5 } & 100 & $0 / 4$ \\
\hline & 100000 & $3 / 4(1872,966,742)$ \\
\hline \multirow[t]{2}{*}{ \#6 } & 100 & $0 / 4$ \\
\hline & 100000 & $0 / 4$ \\
\hline \multicolumn{3}{|c|}{ Vector-hMSCs } \\
\hline \#1-6 & 100 & $0 / 12$ \\
\hline$\# 1$ & 100000 & $0 / 4$ \\
\hline$\# 2$ & 100000 & $2 / 4(1560,540)$ \\
\hline \#3 & 100000 & $1 / 4(874)$ \\
\hline$\# 4$ & 100000 & $0 / 4$ \\
\hline$\# 5$ & 100000 & $0 / 4$ \\
\hline \#6 & 100000 & $0 / 4$ \\
\hline
\end{tabular}

that the former showed TERT expression (Fig. 6E), while the latter did not (Fig. 6I).

\section{Discussion}

hMSCs maintain the homeostasis of bone and cartilage and are found in adult human BM. Those obtained from human in late adulthood still exhibit osteogenic potency (15). Thus, it is thought that hMSCs maintain lifetime self-renewal and differentiation capacity in vivo. However, several previous studies showed that the self-renewal potency of hMSCs is decreased by long-term culture in vitro (16-18) and that FGF-2 expands multipotent hMSCs with high proliferation potential (7). It is also possible that cellular senescence in hMSCs, which might be induced by TGF- $\beta 1$ and increased levels of CDK inhibitors $\left(\mathrm{p} 16^{\text {INK4a }}, \mathrm{p} 21^{\text {Cip1 }}\right.$, and p53), is reversed by FGF-2 $(19,20)$. In the present study, six $\mathrm{FGF}(+)$ hMSC clones were cultureable for more than one year. The mechanism by which they were immortalized is still unknown, but these cell lines retain mutipotential differentiation activity. Thus, these human BM-derived MSCs can be cultured long-term in vitro, without losing their peculiar morphological, phenotypical, and functional characteristics and might become a universal source of cells for regenerative therapies. To elucidate the mechanism of immortalization, we analyzed telomere and telomerase in these immortalized hMSCs. Long telomere lengths (approximately $40 \mathrm{~kb}$ ) were maintained without detectable telomerase activity. 


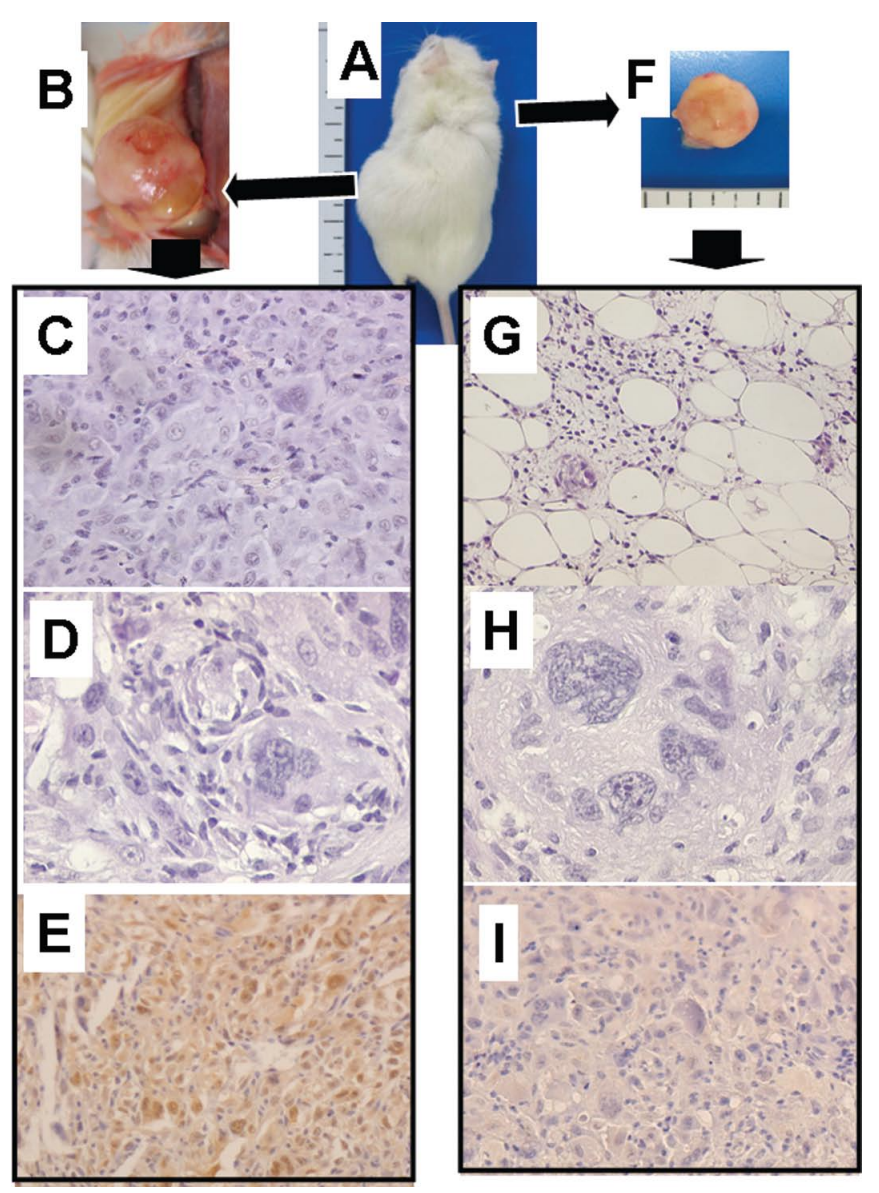

Figure 6. Tumor formation in NOD/SCID mice. (A), Subcutaneous transplantation of $10^{2}$ TERT-transfectedhMSC\#3 (leftside) and $10^{5}$ vector-transfected hMSC\#3 (right side) in NOD mice. (B), Subcutaneous transplantation of TERT-transfected hMSC\#3 (10 ${ }^{2}$ cells) developed large tumor formation. (C), Histological findings of this tumor showed teratocarcinoma without differentiated cells. (D), In this tumor, vascular invasion and polynuclear cells were determined. (E), Immunohistological examination of TERT revealed that most tumor cells were positive. (F), In contrast, subcutaneous transplantation of vector-transfected hMSC\#3 ( $10^{5}$ cells) developed a soft tumor. ( $\mathrm{G}$ and $\mathrm{H}$ ), This tumor showed teratoma with differentiated cells such as adipose and cartilage. (I), This tumor did not contain TERT expressing cells.

Thus, telomeres in these cells are maintained by ALT, which is characterized phenotypically by long and heterogeneous telomeres. ALT-activated tumors, relatively low in human malignancies, are sometimes of mesenchymal origin, and there is evidence to suggest an MSC origin for ALT immortalization in cell lines (21). Therefore, ALT activation of these hMSCs might be characteristic of the mesenchymal origin cells.

Telomerase activity and TERT expression were repressed in our hMSCs clones, even after the cells acquired immortalization. Their repression occurred in ALT-activated hMSCs, although there were several instances of hMSCs with telomerase activation to maintain telomere length for acquiring immortalization $(20,22)$. Thus, two mechanisms based on the presence of either telomerase or ALT expression influence hMSC immortalization. Several reports of hMSC immortalization via TERT transduction, and the present data describing immortalization in FGF-2expanded hMSCs via an ALT mechanism, support this discrimination. However, the mechanism of these activation on hMSCs in vivo remains unknown and the effects of both factors at the same time on hMSCs are unclear.
In the present study, human BM-derived MSCs could be cultured long-term in medium with FGF-2 without losing their peculiar morphological, phenotypical, and functional characteristics. TERT-transfected hMSCs retain the potential of chondrogenic, osteogenic, and adipogenic differentiation but display an increased capacity of colony formation in soft agar and increased tumorigenicity in immunodeficient mice. The transduction of TERT obviously induces the malignant phenotypes of hMSCs, which can also be cultured long-term in vitro, without loss of their morphological, phenotypical, and functional characteristics. In these TERT-transfected clones, hMSCs that express TERT exhibit malignant phenotypes, while those that lose TERT expression do not. Therefore, TERT transduction might play a significant role in the malignant transformation of hMSCs. Although telomerase activity was upregulated in TERT-transfected clones, telomere lengths were as long as those in ALT-activated clones before TERT transfection, indicating that transduced TERT might play a role in malignant transformation other than telomere maintenance. Recent reports suggest that TERT is active in a wide variety of functions, and is a component of RNAdependent RNA polymerase (23). Therefore, activation of TERT might correlate with malignant transformation of hMSCs. TERT-transduced hMSCs also have extended replicative capacity while maintaining their differential capacity $(22,24)$. Therefore, since our hMSCs were immortalized by ALT after FGF-2 treatment before TERT transfection, other factors might be necessary for malignant transformation of normal hMSCs.

In conclusion, human BM-derived MSCs can be cultured long-term in vitro by ALT telomere maintenance without losing their morphological, phenotypical, and functional characteristics. Moreover, these cells are malignantly transformed by TERT transduction, suggesting that activation of TERT is correlated with cancer formation of hMSCs. These cells might be a model for cancer stem cells if they derive from normal tissue stem cells. Further studies are necessary to fully understand the mechanism of malignant transformation by TERT transduction and any correlation with cancer stem cells. Our results support the concept that the biological properties of hMSCs after in vitro expansion remain suitable for use in cell therapy approaches; however, considering the interest in the utilization of hMSCs in several fields of medicine and the potential risk of developing malignant transformation during the expansion period, we strongly recommended the establishment of a suitable test for phenotypic, functional, and genetic characteristics of hMSCs after in vitro expansion to further guarantee the safety of the patient.

\section{Acknowledgements}

We thank Professor M. Ochi and other surgeons and orthopedic surgeons in Graduate School of Biomedical Sciences, Hiroshima University for providing clinical support for this study. This research was partially supported by a Grant-in-Aid for Scientific Research (B) (Nos. 19390449. 21390474 and 22700917) and (Hoga) (19659324 and 20659198) from the Ministry of Education, Culture, Sports, Science, and Technology and those from the Ministry of Health, Labor, and Welfare of the Government of Japan. 


\section{References}

1. Okamoto T, Aoyama T, Nakayama T, Nakamata T, Hosaka T, Nishijo K, Nakamura T, Kiyono T and Toguchida J: Clonal heterogeneity in differentiation potential of immortalized human mesenchymal stem cells. Biochem Biophys Res Commun 295: 354-361, 2002.

2. Takeda Y, Mori T, Imabayashi H, Kiyono T, Gojo S, Miyoshi S, Hida N, Ita M, Segawa K, Ogawa S, Sakamoto M, Nakamura S and Umezawa A: Can the life span of human marrow stromal cells be prolonged by bmi-1, E6, E7, and/or telomerase without affecting cardiomyogenic differentiation? J Gene Med 6: 833-845, 2004

3. Mori T, Kiyono T, Imabayashi $\mathrm{H}$, Takeda $\mathrm{Y}$, Tsuchiya $\mathrm{K}$, Miyoshi S, Makino H, Matsumoto K, Saito H, Ogawa S, Sakamoto M, Hata J and Umezawa A: Combination of hTERT and bmi-1, E6, or E7 induces prolongation of the life span of bone marrow stromal cells from an elderly donor without affecting their neurogenic potential. Mol Cell Biol 25: 5183-5195, 2005.

4. Saito M, Handa K, Kiyono T, Hattori S, Yokoi T, Tsubakimoto T, Harada H, Noguchi T, Toyoda M, Sato S and Teranaka T: Immortalization of cementoblast progenitor cells with Bmi-1 and TERT. J Bone Miner Res 20: 50-57, 2005.

5. Terai M, Uyama T, Sugiki T, Li XK, Umezawa A and Kiyono T: Immortalization of human fetal cells: the life span of umbilical cord blood-derived cells can be prolonged without manipulating p16INK4a/RB braking pathway. Mol Biol Cell 16: 1491-1499, 2005 .

6. Tsutsumi S, Shimazu A, Miyazaki K, Pan H, Koike C, Yoshida E, Takagishi K and Kato Y: Retention of multilineage differentiation potential of mesenchymal cells during proliferation in response to FGF. Biochem Biophys Res Commun 288: 413-419, 2001.

7. Yanada S, Ochi M, Kojima K, Sharman P, Yasunaga Y and Hiyama E: Possibility of selection of chondrogenic progenitor cells by telomere length in FGF-2-expanded mesenchymal stromal cells. Cell Prolif 39: 575-584, 2006.

8. Hiyama E, Yokoyama T, Hiyama K, Michio Y, Santo T, Kodama T, Ichikawa T and Matsuura Y: Alteration of telomeric repeat length in adult and childhood solid neoplasias. Int $\mathrm{J}$ Oncol 6: 13-16, 1995.

9. Hiyama K, Ishioka S, Shirotani Y,Inai K, Hiyama E, Murakami I, Isobe T, Inamizu T and Yamakido M: Alterations in telomeric repeat length in lung cancer are associated with loss of heterozygosity in p53 and Rb. Oncogene 10: 937-944, 1995.

10. Kim NW, Piatyszek MA, Prowse KR, Harley CB, West MD Ho PLC, Coviello GM, Wright WE, Weinrich SL and Shay JW: Specific association of human telomerase activity with immortal cells and cancer. Science 266: 2011-2015, 1994.

11. Piatyszek MA, Kim NW, Weinrich SL, Hiyama K, Hiyama E, Wright WE and Shay JW: Detection of telomerase activity in human cells and tumors by a telomeric repeat amplification protocol (TRAP). Methods Cell Sci 17: 1-15, 1995.

12. Chromczynski P and Sacchi N: Single-step method of RNA isolation by acid guanidinium thiocyanate-phenol-chloroform extraction. Anal Biochem 162: 156-159, 1987.
13. Hiyama E, Hiyama K, Shay JW and Yokoyama T: Immunohistochemical detection of telomerase (hTERT) protein in human cancer tissues and a subset of cells in normal tissues. Neoplasia 3: 17-26, 2001.

14. Pittenger MF, Mackay AM, Beck SC, Jaiswal RK, Douglas R, Mosca JD, Moorman MA, Simonetti DW, Craig S and Marshak DR: Multilineage potential of adult human mesenchymal stem cells. Science 284: 143-147, 1999.

15. Radio NM, Doctor JS and Witt-Enderby PA: Melatonin enhances alkaline phosphatase activity in differentiating human adult mesenchymal stem cells grown in osteogenic medium via MT2 melatonin receptors and the MEK/ERK (1/2) signaling cascade. J Pineal Res 40: 332-342, 2006.

16. Jaiswal N, Haynesworth SE, Caplan AI and Bruder SP: Osteogenic differentiation of purified, culture-expanded human mesenchymal stem cells in vitro. J Cell Biochem 64: 295-312, 1997.

17. Deans RJ and Moseley AB: Mesenchymal stem cells: biology and potential clinical uses. Exp Hematol 28: 875-884, 2000.

18. Jiang Y, Jahagirdar BN, Reinhardt RL, Schwartz RE, Keene CD, Ortiz-Gonzalez XR, Reyes M, Lenvik T, Lund T, Blackstad M, Du J, Aldrich S, Lisberg A, Low WC, Largaespada DA and Verfaillie CM: Pluripotency of mesenchymal stem cells derived from adult marrow. Nature 418: 41-49, 2002.

19. Bianchi G, Banfi A, Mastrogiacomo M, Notaro R, Luzzatto L, Cancedda $R$ and Quarto R: Ex vivo enrichment of mesenchymal cell progenitors by fibroblast growth factor 2. Exp Cell Res 287: 98-105, 2003

20. Zhang X, Soda Y, Takahashi K, Bai Y, Mitsuru A, Igura K, Satoh H, Yamaguchi S, Tani K, Tojo A and Takahashi TA: Successful immortalization of mesenchymal progenitor cells derived from human placenta and the differentiation abilities of immortalized cells. Biochem Biophys Res Commun 351: 853-859, 2006.

21. Lafferty-Whyte K, Cairney CJ, Will MB, Serakinci N, Daidone MG, Zaffaroni N, Bilsland A and Keith WN: A gene expression signature classifying telomerase and ALT immortalization reveals an hTERT regulatory network and suggests a mesenchymal stem cell origin for ALT. Oncogene 28: 3765-3774, 2009.

22. Simonsen JL, Rosada C, Serakinci N, Justesen J, Stenderup K, Rattan SI, Jensen TG and Kassem M: Telomerase expression extends the proliferative life-span and maintains the osteogenic potential of human bone marrow stromal cells. Nat Biotechnol 20: 592-596, 2002.

23. Maida Y, Yasukawa M, Furuuchi M, Lassmann T, Possemato R, Okamoto N, Kasim V, Hayashizaki Y, Hahn WC and Masutomi K: An RNA-dependent RNA polymerase formed by TERT and the RMRP RNA. Nature 461: 230-235, 2009.

24. Shi S, Gronthos S, Chen S, Reddi A, Counter CM, Robey PG and Wang CY: Bone formation by human postnatal bone marrow stromal stem cells is enhanced by telomerase expression. Nat Biotechnol 20: 587-591, 2002. 